\title{
Knowledge \& Utilization of Long Lasting Impregnated Nets among Housewives in Al Moneera City and Al Koozi Villages, Hodieda Governorate, Republic of Yemen 2007
}

\author{
Nahid A. Baktayan ${ }^{1 *}$, Ali M. Assabri², Belquis A. Farea ${ }^{3}$, Arwa A. Farea ${ }^{4}$ \\ ${ }^{1}$ Community Medicine, Faculty of Medicine \& Health Sciences, Aden University, Aden, Yemen \\ ${ }^{2}$ Community Medicine, Faculty of Medicine \& Health Sciences, Sana'a University, Sana'a, Yemen \\ ${ }^{3}$ National Malaria Control Program, Ministry of Public Health \& Population, Sana'a, Yemen \\ ${ }^{4}$ Faculty of Pharmacy, University of Science and Technology, Sana'a, Yemen \\ Email: ^nbaktayan@gmail.com
}

How to cite this paper: Baktayan, N.A., Assabri, A.M., Farea, B.A. and Farea, A.A. (2021) Knowledge \& Utilization of Long Lasting Impregnated Nets among Housewives in $\mathrm{Al}$ Moneera City and $\mathrm{Al} \mathrm{Koozi}$ Villages, Hodieda Governorate, Republic of Yemen 2007. Health, 13, 306-322.

https://doi.org/10.4236/health.2021.133025

Received: January 29, 2021

Accepted: March 23, 2021

Published: March 26, 2021

Copyright $\odot 2021$ by author(s) and Scientific Research Publishing Inc. This work is licensed under the Creative Commons Attribution International License (CC BY 4.0).

http://creativecommons.org/licenses/by/4.0/

\begin{abstract}
Malaria is considered as one of the main health problem in the Republic of Yemen. In February 2006 National Malaria Control Program started freely distribution of Long Lasting Impregnated Nets (LLINs) for vulnerable groups to malaria infection (pregnant women and children under 5 years). After one year of distribution, there is a need to measure the use rate among vulnerable groups and explore factors affecting their use. The aim of the study was to investigate the use rate of long lasting impregnated nets among children under five years and pregnant women in $\mathrm{Al}$ Moneera city and $\mathrm{Al}$ Koozi villages of Hodieda governorate. Methodology: Cross section study with multistage sample, Random selection of one urban and one rural locality. 768 housewives were interviewed, proportionally allocated sample according to the number of vulnerable groups in each locality. Systematic random sample to select households. Results: The use rate of LLINs for vulnerable groups was 74.3\% CI (71.2 - 77.4). There was no statistically significant association between the use of LLINs and residence, also with ages of housewives, occupation, type of house, but there is strong statistically significant association between use of LLINs and having correct information about how to use and to take care of them $\mathrm{P}$ value $<0.001$, also with educational status of housewives; $\mathrm{P}$ value 0.03 and educational status of fathers; $\mathrm{P}$ value 0.01 . (84\%) have correct information about how to use and take care of LLINs and the main source of information was health workers who distributed them. Only $13.4 \%$ realized pregnant women as a vulnerable group to malaria infection while
\end{abstract}


about $77.9 \%$ realized children as a vulnerable group to malaria infection. The main reason of LLINs use (84.8\%) was protection from nuisance of insects. Conclusion and recommendations: There is shortage in the information about the protective effect of LLINs from malaria infection and the main reason of using them is protection from nuisance of insects. There is a need to increase health awareness about the role of LLINs in malaria prevention.

\section{Keywords}

Vulnerable Groups to Malaria Infection, Long-Lasting Impregnated Nets (LLINs) Use Rate, Housewives, Al Moneera City and Al Koozi Villages, Hodieda Governorate, Yemen

\section{Introduction}

Malaria is a common, serious and sometimes fatal tropical disease. In the East Mediterranean Region, reported malaria cases about 2.7 million in 2005 represent only a fraction of true incidence. It is estimated that about 10 million malaria episode and 49,000 malaria related deaths occur every year in the region. Out of which 95\% are believed to occur in six countries: Afghanistan, Djibouti, Pakistan, Somalia, Sudan and Yemen [1].

In nearly all situations, vector control is the main measure to prevent malaria transmission. World Health Organization (WHO) recommends Integrated Vector Management (IVM) as a systematic approach to select the best method of vector control or combination of methods to reduce transmission. InsecticideTreated Nets (ITNs) prevent malaria transmission and child deaths [2]. A new kind of net, which retains its insecticidal properties without needing frequent re-treatment, has recently been developed. These Long-Lasting Impregnated Nets (LLINs) work for four to five years. It has been estimated that about 30 million nets per year are required to meet the target of having $60 \%$ of at-risk populations' young children and pregnant women in Africa, protected by nets within three years [3].

Malaria is considered as one of the main health problem in the Republic of Yemen. Regarding malaria Yemen is epidemiologically classified as Afro-tropical because of the predominance of the life threatening species the Plasmodium Falciparum, which accounts for over $90 \%$ of the recorded malaria cases annually, and predominance of the potent malaria vector Anopheles Arabiensis, which is one of the most effective malaria vectors all over the world except in Socotra Island and part of Al-Mahara governorate which is epidemiologically classified as oriental with the predominant of less effective vector Anopheles Culicifacies and the species is Plasmodium Vivax [4].

Republic of Yemen is divided from epidemiological point of view in to four regions:

1) The coastal plain (Tihama region) from the sea level up to 200 meter eleva- 
tion.

2) The foothills and middle highest: 200 - 2000 meters elevation.

3) The central highlands: from 2000 meters elevation and above.

4) The arid slopes from the plateaus to Arabian Desert [5].

The endemicity differs from one area to another varying from hypo to meso and hyperendemicity [6]. Out of population $60 \%$ live under the risk of malaria infection [7]. The transmission season is from October to March along the coastal areas and from April to September/October in the foothills and the mountains below 1500 meters height [7].

The Tihama plain is considered the most malarias area in the Republic of Yemen. The steady expansion of agriculture projects in this area has contributed to the increase in breeding of vectors of malaria. Moreover, there are five main valleys (Maor, Surdud, Siham, Zabid, and Rima) passing through Tihama plain that are also natural breeding places [8].

One of the objectives of National Malaria Control Program (NMCP) is to protect $60 \%$ of pregnant women and children under 5 years by LLINs by the year 2010. LLINs are expected not only to protect from malaria but also from other vector borne diseases (VBDs) like Leishmaniasis, yellow fever, Dengue fever etc. [9].

In February 2006 The NMCP started to distribute LLINs in five districts of Hodieda governorate and Taiz city in Taiz governorate. They targeted the vulnerable groups the children under five years and women in reproductive age. The distributed LLINs are free of charge funded by (Global Fund for fighting HIV/AIDS, Tuberculosis and Malaria) GFATM. The LLINs are PermaNets ${ }^{\circ}$ which have been tested by an international reference laboratory according to WHO protocols. LLINS are rectangular of family size $190 \times 180 \times 150 \mathrm{~cm}, 100 \%$ polyester, durable and non-flammable. The insecticide $55 \mathrm{mg} / \mathrm{m}^{2}$ deltamethrin. Yarns 100 deniers $40 \mathrm{~g} / \mathrm{m}^{2}$. Mesh $165 / \mathrm{inch}^{2}=25 / \mathrm{cm}^{2}$ [10]. The first stage of freely distributed LLINs was in Hodieda governorate. There is some variation in malaria incidence in the Republic of Yemen, $25 \%$ of malaria cases occur in Hodieda governorate, Annual parasite incidence API (54/1000) [11].

\subsection{Rationale of the Study}

- Knowledge of the etiology, methods of transmission and prevention of malaria play a role in health-seeking behavior and prevention in relation to malaria bednets utilization.

- Proper use and care of bednets reduce malaria health risk especially to vulnerable groups targeted by bednets (pregnant women and children under five years).

- After one year of freely distribution of LLINs in malaria endemic area (Hodieda governorate), there is a need to measure the use rate among vulnerable groups to malaria infection especially in the season of malaria transmission and to study factors affecting their use.

- Lack of data about utilization of bed nets in Yemen. 


\subsection{Aim of the Study}

To investigate the use rate of Long Lasting Impregnated Nets (LLINs) among children under five years and pregnant women in Al Moneera city and Al Koozi villages of Hodieda governorate (west of Republic of Yemen), and to explore knowledge attitude and practices of housewives towards LLINs in malaria prevention among these vulnerable groups.

\subsection{Specific Objectives}

1) To measure use rate of (LLINs) among vulnerable groups to malaria infection (children under five years \& pregnant women) in the study areas.

2) To assess knowledge of housewives towards malaria infection.

3) To assess knowledge, attitude and practices of housewives in study areas towards (LLINs) in malaria prevention among vulnerable groups to malaria infection.

4) To identify the effect of certain demographic, socio-economic factors on the use of LLINs in preventing malaria among vulnerable groups

5) To compare between one urban locality (Al Moneeracity) and one rural locality (Al Koozi villages) regarding using of LLINs.

\section{Materials and Methods}

\subsection{Study Area}

Hodieda governorate is situated in the Tihama at the west of the Republic of Yemen, which is, consider as the main malarias area in the Republic of Yemen [10].

\subsection{Study Area Population}

The total population is about $2,161,379$ inhabitant [12]. Children under five years represent $15 \%$, pregnant women $3.8 \%$ [12].

\subsection{Study Design}

Cross-section study.

The target population: Housewives of selected households have been interviewed.

We measured the use rate of LLINs:

$$
\text { LLINs use rate }=\frac{\text { Users }}{\text { Target population }} \times 100
$$

(The target population is vulnerable groups of the households in the sample who received LLINs from NMCP at the study area).

\subsection{Sample Size}

A sample was calculated by the following formula:

$$
n=p q z^{2} / d^{2}=384
$$


A large sample size was needed because of design effect [13], since we will use multistage sample, (Urban-Rural). A design effect (DEFF) was estimated as 2. Therefore the calculated sample size was multiplied by 2 . $n=768$.

Sampling method: Multistage sampling technique has been used.

1) Purposive selection of Hodieda governorate as the main malaria endemic area, and NMCP distributed LLINs in some of its districts.

2) Random selection of one urban and one rural locality from districts targeted by LLINs distribution in Al-Hodieda governorate,, Al Moneera city in Al Moneera district was an urban locality in our study, and Al-Koozi villages in Al-Qanawis district was randomly selected from the list of rural localities of targeted districts.

3) Proportionally allocated sample was used. The sample distribution is shown in Table 1.

4) We used Systematic random sample to select households. We calculated Sampling interval as the following: Sample interval $=$ Number of households/ Sample size $\times 100$.

We selected first household randomly then each fourth household in Al Moneera city and each fifth household in Al Koozi villages. In random selection, if the selected household not received LLINs, we moved to the next household.

\section{Selection criteria of study population:}

Households from Al Moneera city and Al-Koozi villages who received LLINs from NMCP and have pregnant women and/ or children under five years

\subsection{Data Collection Technique}

15 educated women from the same localities (Al Moneera city and Al Koozi villages) were trained to perform the interviews with targeted sample housewives. We trained data collectors to check the existence of LLINs by themselves (visual check). Collection of data was conducted in the first two weeks of February 2007.

\subsection{Data Collection Tool}

A structured questionnaire in Arabic language was designed by the author based on the study objectives. It contained 31 questions; it included three items, the first one about socio-demographic data, the second item about knowledge, and the third one about attitudes and practices. A pilot study was conducted before the implementation of the study in the same localities urban and rural, 20 questionnaires from Al Moneera city, and 30 questionnaires from Al Koozi villages.

Table 1. The distribution of sample in study area.

\begin{tabular}{cccc}
\hline Study area & targeted population & Percent & Sample size \\
\hline Al Moneera & 2972 & $35 \%$ & 269 \\
Al-Koozi & 5618 & $65 \%$ & 499 \\
Total & 8590 & $100 \%$ & 768 \\
\hline
\end{tabular}

*Women in reproductive age \& Children under 5 years old. 
The questionnaire had been modified based to the result of pilot study.

\subsection{Ethical Considerations}

Before the implementation of the study, approval was obtained from the health office authority, local council in the targeted districts and from office of NMCP in Tihama region. The housewives were informed about the objectives of the study, and informed that no names will be written in the questionnaire in order to ensure the confidentiality of the information they provided. Verbal consent was obtained from every one enrolled in the study.

\subsection{Statistical Analysis}

The data was coded to be suitable for computer feeding. The statistical analysis was performed using computer program, statistical package for social science (SPSS version 13).

\section{Results}

\subsection{Characteristics of Study Population}

We conducted our study in the first two weeks of February 2007 (the season of malaria transmission in study area). The total number of study population was 768, from Al Moneera city 269, and 499 from Al-Koozi villages. More than half of them in the age group (20 - 30 year). The mean age of study population is 28.4 \pm 7.1 year.

97.0\% of study population are housewives. Out of 768 of study population only 127 of them (16.5\%) were pregnant, and 760 of households (99\%) have children under 5 years.

After one year of LLINs distribution, $98 \%$ of those who received LLINs still have them in place, only $2 \%$ were lost.

\subsection{Knowledge about Malaria \& LLINs}

Regarding knowledge about route of malaria transmission, $65.2 \%$ said mosquito bite. $84 \%$ of study population mentioned high temperature as a symptom of malaria infection. Only $31.1 \%$ of study population mentioned convulsion as a complication of malaria infection.

Regarding the source of information about LLINs among study population 79.2\% mentioned health workers who distributed them. Regarding the knowledge of housewives about the protective methods from malaria infection, only $44.4 \%$ of them mentioned LLINs one of the protective method.

The majority of housewives (84\%) have correct information about how to use and take care of LLINs. This is an open end question, we considered the answer is true when they hang LLINs over the bed and cover it completely, and when they keep LLINs out of sun and out of reach of children either put it in specific case or in the cupboard or even hang it over the bed

The majority of housewives (84\%) have correct information about how to use 
and take care of LLINs. This is an open end question, we considered the answer is true when they hang LLINs over the bed and cover it completely, and when they keep LLINs out of sun and out of reach of children either put it in specific case or in the cupboard or even hang it over the bed

Figure 1 shows the correct information of housewives about how to use and care of LLINs.

\subsection{Attitudes \& Practices}

More than three quarters of study population (77.1\%) believe that LLINs have a protective effect from malaria infection, while $4.4 \%$ believe that LLINs have no any protective effect and $18.5 \%$ do not know.

The perception towards the protective effect of LLINs from malaria infection which was mentioned by 592 (77.1\%) differ according to age groups, out of those who believe that LLINs have a protective effect (53.7\%) in age groups (20 - 30 years).

The perception about the protective effect of LLINs is more in illiterate women than others $69 \%$.

Regarding perception of study population about vulnerable groups to malaria infection, regarding perception of study population about vulnerable groups to malaria infection, more than three quarter of housewives $77.9 \%$ mentioned children, but only $13.4 \%$ mentioned pregnant women. Detailed description in Figure 2.

\section{Utilization of LLINs among vulnerable groups to malaria infection}

The use rate of LLINs in general for vulnerable groups, nearly three quarters of households (74.3\%), use it CI (71.2 - 77.4). Figure 3, but regarding use rate of LLINs at the previous night of interview for children under five years only (51.9\%) CI (48.4 - 55.4) and for pregnant women (59.3\%), CI (55.8 - 62.8).

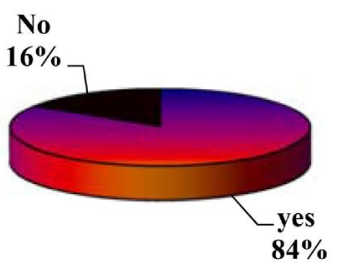

Figure 1. Correct Information of housewives about how to use and care of LLINs.

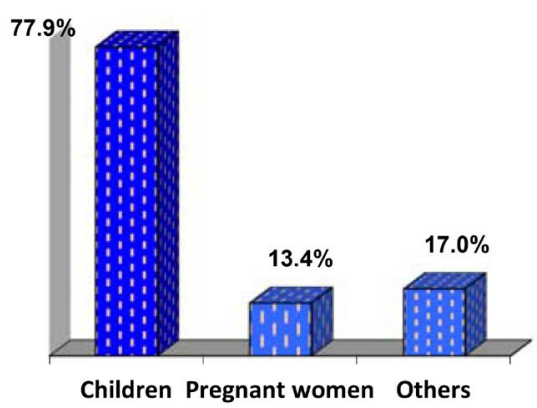

Figure 2. Perception of housewives about vulnerable groups to malaria infection. 


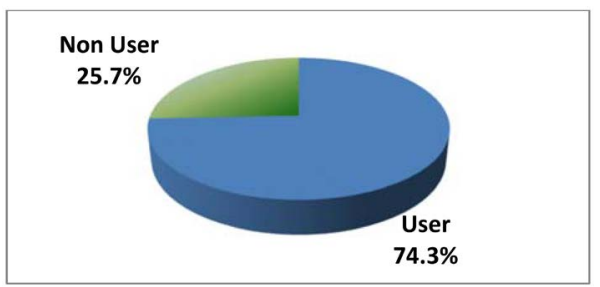

Figure 3. Use rate of LLINs among vulnerable groups.

In regards to the reasons of using LLINs $84.8 \%$ mentioned protect from nuisance of insects and only $29.3 \%$ mentioned protect from malaria. On the other hand for reasons of not using LLINs $23.7 \%$ mentioned there are no mosquito and other insects, $19.6 \%$ mentioned LLINs need fixation.

For time of sleeping of children under 5 years, more than three quarters (79.1\%) sleep at 8 o'clock P.M, while (18.4\%) at 7 o'clock P.M and only (2.5\%) after 10 o'clock P.M. On the other hand time of sleeping of pregnant women; out of 127 pregnant women, 72 of them $56.7 \%$ sleep after 10 o'clock P.M, while 55 pregnant women $(43.3 \%)$ at (8 - 9 o'clock P.M).

For time of using LLINs $67.1 \%$ use LLINs at 8-9 o'clock P.M and $14.8 \%$ after 10 o'clock P.M and $14.4 \%$ at 7 o'clock P.M.

Out of 84 of vulnerable groups to malaria infection who sleep under LLINs after 10 o'clock, $85.7 \%$ are pregnant women only $14.3 \%$ are children under 5 years.

Regarding the number of persons who sleep under one LLIN; mean of persons sleep under one LLIN is $2.5 \pm 1$ person for each LLIN, minimum number 1 , maximum number 6.

At time of study $99.8 \%$ of study population sleep indoor, only $0.2 \%$ sleep outdoor.

In regards to take care of LLINs, $57.1 \%$ keep it out of children, $43.5 \%$ keep it out of sun.

With Regards to the place of keeping LLINs, we found $54.0 \%$ hang it over the bed, $26.1 \%$ put it in its specific case, $18.2 \%$ in the cupboard, $1.2 \%$ leave it in its place, and only $0.5 \%$ put it under the sun. Regarding other persons beside vulnerable groups sleep under LLINs $64.5 \%$ said no, only $35.5 \%$ said yes. With regards to action with torn LLINs $73.6 \%$ sew it, $13.4 \%$ leave it and $13.0 \%$ don't know any action. For the number of times of washing LLINs $56.8 \%$ did not wash it and 31.6 washed it one time.

\subsection{Relationship between Using of LLINs and Study Variables}

Regarding the relationship between age groups of study population and using of LLINs in general among vulnerable groups to malaria infection, we found that the use rate in younger age groups was more than older one, but there is no statistical significant association (Table 2).

There were some differences between type of occupation of study population and the using of LLINs, but these differences are not statistically significant 
(Table 3).

There were some differences between type of houses and the using of LLINs, but they are not statistically significant (Table 4).

The using of LLINs in urban area was more than in rural areas, but the difference is not statistically significant (Table 5).

The study of the relationship between income of households and using of LLINs illustrates that there are some difference in using with different income

Table 2. The relationship between Age groups of the study population and using of LLINs.

\begin{tabular}{cccc}
\hline Age group & Total & Users & Use rate \\
\hline$<20$ & 78 & 65 & $83.0 \%$ \\
$20-30$ & 426 & 319 & $75.0 \%$ \\
$31-40$ & 211 & 155 & $73.0 \%$ \\
$>40$ & 53 & 34 & $64.0 \%$ \\
Total & 768 & 571 & $74 \%$ \\
\hline
\end{tabular}

$P$ value $=0.09 ; \chi^{2}=6.35$.

Table 3. The relationship between occupation of study population and using of LLINs.

\begin{tabular}{cccc}
\hline Occupation & Total & Users & Use rate \\
\hline Housewife & 745 & 554 & $73.4 \%$ \\
Employee & 9 & 6 & $66.7 \%$ \\
Farmer & 8 & 6 & $75.0 \%$ \\
Student & 6 & 5 & $83.3 \%$ \\
Total & 768 & 571 & $74.4 \%$ \\
\hline
\end{tabular}

P value $0.91 ; \chi^{2}=0.53$

Table 4. The relationship between type of house of study population and using of LLINs.

\begin{tabular}{cccc}
\hline Type of house & Total & Users & Use rate \\
\hline Hut & 147 & 102 & $69.4 \%$ \\
Clay & 33 & 22 & $66.7 \%$ \\
Block & 588 & 447 & $76.0 \%$ \\
Total & 768 & 571 & $74.4 \%$ \\
\hline
\end{tabular}

P value $=0.15 ; \chi^{2}=3.78$

Table 5. The relationship between residence of study population and LLINs use rate.

\begin{tabular}{cccc}
\hline Residence & Total & Users & Use rate \\
\hline Urban & 269 & 206 & $76.6 \%$ \\
Rural & 499 & 365 & $73.1 \%$ \\
Total & 768 & 571 & $74.4 \%$ \\
\hline
\end{tabular}

$\mathrm{P}$ value $=0.29 ; \chi^{2}=1.0$. 
but it is not statistically significant (Table 6).

The relationship between having correct information about how to use and care of LLINs and using them indicates that, there is strong statistical significant association, $\mathrm{P}$ value $<0.001$ and $\chi^{2}=72.9$. Housewives who have correct information use them more than those who have no correct information (Table 7).

The relationship between educational status of study population and use of LLINs illustrates that, the using of LLINs was associated with educational status. Generally, high level of education leads to increase in the using of LLINs, the difference is statistically significant, $\mathrm{P}$ value is 0.03 , although the use rate in higher education was less than others, this can be interpreted due to small sample size of that stratum (Table 8).

The study of the relationship between educational status of the fathers and Table 6. The relationship between income of households and LLINs use rate.

\begin{tabular}{cccc}
\hline Monthly income in Yemeni Riyal & Total & Users & Use rate \\
\hline$<10,000$ & 301 & 225 & $74.8 \%$ \\
$10,000-20,000$ & 312 & 227 & $72.8 \%$ \\
$>20,000-<30,000$ & 116 & 91 & $78.4 \%$ \\
$30,000-40,000$ & 34 & 25 & $73.5 \%$ \\
$>40,0001$ & 5 & 3 & $60 \%$ \\
Total & 768 & 571 & $74.4 \%$ \\
\hline
\end{tabular}

P value $=0.73 ; \chi^{2}=2.01$

Table 7. The relationship between having correct information about how to use and care of LLINs and using of them.

\begin{tabular}{cccc}
\hline Having correct information about LLINs & Total & Users & Use rate \\
\hline Have correct information about how to use and care of LLINs & 645 & 519 & $80.5 \%$ \\
Have no correct information about how to use and care of LLINs & 123 & 54 & $44 \%$ \\
Total & 768 & 573 & $74.6 \%$ \\
\hline
\end{tabular}

$P$ value $<0.001$ and $\chi^{2}=72.9$.

Table 8. The relationship between educational status of study population and using of LLINs.

\begin{tabular}{cccc}
\hline Educational status & Total & Users & Use rate \\
\hline Illiterate & 550 & 403 & $73.3 \%$ \\
Read \& write & 99 & 64 & $73.6 \%$ \\
Primary & 76 & 66 & $75.0 \%$ \\
Secondary & 36 & 35 & $97.2 \%$ \\
Higher & 7 & 5 & $71.4 \%$ \\
Total & 768 & 573 & $74.6 \%$ \\
\hline
\end{tabular}

$P$ value $=0.03 ; \chi^{2}=10.3$ 
Table 9. The relationship between educational status of the fathers and using of LLINs.

\begin{tabular}{cccc}
\hline Educational status of fathers & Total & Users & Use rate \\
\hline Illiterate & 384 & 273 & $71.1 \%$ \\
Read \& write & 89 & 64 & $72.0 \%$ \\
Primary & 154 & 115 & $74.7 \%$ \\
Secondary & 96 & 82 & $85.4 \%$ \\
Higher & 45 & 39 & $86.7 \%$ \\
Total & 768 & 571 & $74.4 \%$ \\
\hline
\end{tabular}

$\mathrm{P}$ value $=0.01 ; \chi^{2}=12.2$.

using of LLINs confirm the statistical significant association, generally there is an increase in the using of LLINs with the increase of level of education of the fathers (Table 9).

\section{Discussion}

Our study revealed that the use rate of LLINs in general among vulnerable groups was (74.3\%), CI (71.2 - 77.4) as represented by means in Figure 1. The use rate of LLINs at the previous night for children under five years only (51.9\%) CI (48.4 - 55.4) and for pregnant women (59.3\%), CI (55.8 - 62.8). Our results illustrated that the use rate of bed nets was more than that in other studies. In rural Kenya, the use rate of bed nets was only $15 \%$ among children under five years [14]. Other study in Uganda showed that only $25 \%$ of children were using bed nets for malaria prevention [15]. The third study in Nyamira district-Kenya showed that, there was low use of ITNs (23.8\%) among children [16].

In our study the using of LLINs was not significantly associated with age as tabulated in Table 2 also not associated with occupation of the study population as in Table 3, type of house as in Table 4, residence (urban and rural) as in Table 5, and households income as in Table 6, but there is a great association with having a correct information about how to use and care of LLINs as represented in Table 7, the using of LLINs was higher among those who have correct information $\mathrm{P}$ value $<0.001, \chi^{2} 72.9$ another statistical association of LLINs using with educational status of the study population as presented in Table 8, high educational status of study population leads to increase of the using of LLINs, $\mathrm{P}$ value 0.03 and $\chi^{2} 10.3$. Also there was a significant association between the educational status of fathers and using of LLINs as represented in Table 9, high educational status of fathers leads to increase using of LLINs, P value 0.01 and $\chi^{2}$ 12.2. The same relation between educational status and using of bed nets was found in two studies, one in rural Kenya, it showed that using of bed nets was affecting with mother's education, $32.7 \%$ of mothers with secondary school study and above use malaria bed nets, While the use rate was only $28.7 \%$ of mothers who complete primary school, $23.9 \%$ of mothers who incomplete primary school and $14.4 \%$ with illiterate mothers [14]. Another study in Nyamira district in Kenya 2003, the possibility of a mother having an insecticide treated 
net was significantly related with the level of education of the mother, occupation and knowledge [16].

There is a positive finding in our study; the majority of households use LLINs in the season of malaria transmission in Tihama region.

On the basis that knowledge about malaria plays a role in health-seeking behavior and prevention in relation to bed nets use, the study illustrated that nearly two-thirds of the study population (65.2\%) identified mosquito bite as a route of malaria transmission, while $(22.9 \%)$ think that malaria is transmitted by drinking dirty water, $(15.4 \%),(5.1 \%)$ think that malaria is transmitted by flies and eat dirty food respectively. But only (48.8\%) of our study population identified mosquito bites as the only route of malaria transmission.

Comparing our study findings with other studies, a study in northern Ghana revealed that $79 \%$ of respondents attributed the cause of malaria to mosquitoes, $24 \%$ due to dirty water (injected by mosquitoes), $5 \%$ due to bad food, $3 \%$ due to housefly and $2 \%$ due to heat/sunshine [17]. But we know that the mosquito is a vector of malaria transmission rather than a causative agent as shown in this study and the following study.

Another study in Burkina Faso showed that $100 \%$ of participants in the focus group discussion know that mosquito bite is causing malaria infection by sucking blood, but $60 \%$ of them mentioned that mosquito cause malaria by deposition of dirty water under the skin, $30 \%$ attributed the cause of malaria to eating dirty food and $20 \%$ to cold, particularly cold rains [18]. From our point of view, the results of the focus group discussion are revealed as statements rather than percents and rates.

Regarding knowledge about malaria symptoms and complications; the majority of our study population (84.9\%) identified high temperature as malaria symptom and $41.8 \%$ mentioned vomiting. One third of study population (33.2\%) don't know any of malaria complications, while $31.1 \%$ mentioned convulsion and $27.2 \%$ mentioned anemia. From our view of point, anemia is more common as a malaria complication rather than convulsion, but the severity of convulsion attracted people more than anemia. There is an increase of knowledge about malaria complications with high educational status.

In a study of northern Ghana, $97 \%$ perceive that high temperature is one of the malaria symptoms, $38 \%$ mentioned vomiting, $27 \%$ chills, $26 \%$ Headache and $8 \%$ loss of appetite [19]. Also a study in Peron, Mali revealed that $95 \%$ of respondents mentioned one symptom of malaria [20]. But in a study of Ugandan children $90 \%$ of caregivers identified fever as a symptom of malaria, $20 \%$ identified convulsion as a sign of severe malaria [15].

Regarding knowledge about LLINs, a high percentage of the study population $84 \%$ have correct information about how to use and care for LLINs, and the main source of information $79.2 \%$ was health workers who distributed them, the information from schools $8.8 \%$, from the community $4.6 \%$ and only $3.7 \%$ from media. In Tanzania's study, $59 \%$ of women mentioned that the source of information about ITNs from media, $28 \%$ from maternal and child health centers and 
$12 \%$ from the community [21]. The high percent in our study mentioned health workers as a source of information comparing other studies because health workers were responsible people for LLINs distribution and they are responsible for people education about LLINs, while in other countries people have to purchase bed nets by themselves.

Regarding attitudes and practices, more than three quarters of Study population (77.1\%) believe that, LLINs have a protective effect against malaria, but in our questionnaire we tried to verify actual knowledge therefore, we included an open end question to explore their knowledge about the protective methods from malaria infection only $44.4 \%$ mentioned LLINs, this indicates that, there is no enough awareness about the role of LLINs as a protective method from malaria infection. More than half of those believe that LLINs have a protective effect from malaria infection, (53.7\%) in age groups (20 - 30 years), but when we reanalyzed this finding and calculated percent of each age group in the study population, we found that there is no difference among age groups and that result because the majority of study population in age group (20 - 30 years). Although our finding showed that the majority of study population believe that LLINs have a protective effect are illiterate, when we reanalyzed this result and calculated percent of each educational status, we found that awareness among illiterates housewives is less than educated one and that result because the majority of study population are illiterates.

Only $13.4 \%$ realize pregnant women as a vulnerable group to malaria infection, but $(77.9 \%)$ mentioned children as a vulnerable group to Malaria infection. From our point of view this result can be explained due to the severity of clinical manifestations of malaria among children.

A study in rural Nigeria illustrated that $76.2 \%$ of respondents perceive that nets could be used to prevent mosquito bites, [22] another study conducted in Tanzania showed that $91 \%$ of participant women believe that using of ITNS is one of the best interventions to protect pregnant women from malaria [21].

In General there is shortness in knowledge about the protective effect of LLINs from malaria infection among vulnerable groups. Our findings revealed that the main reason of using LLINs (84.8\%) was protection from nuisance of insects; only $29.3 \%$ was protection from malaria infection. The similar result was found in a study in northern Ghana in which ITNs were perceived to be of benefit for protection from seasonally abundant nuisance mosquitoes [19]. Regarding the main three reasons of not using LLINs by our study population (62.9\%) were: there is no mosquito and other insects, LLINs need fixation and chemicals cause allergy. The results of a study about malaria in Hajjar valley of Hadramout governorate illustrated that (65\%) of respondents were not using bed nets because they were unavailable and (33\%) because of their high cost [5].

Regarding the time of using LLINs among vulnerable groups, (14.4\%) use LLINs at 7 o'clock P.M, while more than two -third of vulnerable groups (67.1\%) use it at 8 - 9 o'clock P.M, and (3.7\%) use it at day and night. There is no recent study about the feeding time of mosquitoes in the Republic of Yemen. In 1986, 
N. Ahmed and S. Matta described the breeding site and behavior of An. Arabiensis vector in Tihama region; they found that An. Arabiensis bite throughout the night with the peak period of activity between 10.00 P.M to 1:00 A.M, it feeds on human hosts both indoors and outdoors, and rest mainly indoors in both human and animal shelters [23]. According to the findings of our study some pregnant women are still at risk of malaria infection although they use LLINs, because $(85.7 \%)$ of pregnant women who use LLINs, use it after 10 o'clock, therefore the benefit of LLINs use in malaria prevention is relatively low, while only $14.3 \%$ of children under 5 years use LLINs after 10 o'clock therefore, they are at low risk of malaria infection compared with pregnant women.

\section{Limitations of the Study}

The study was implemented in February 2007 after one year of LLINs distribution in five districts in Hodieda governorate, After one year of our study, NMCP distributed LLINs in 21 districts all over the country, therefore another study in other malaria districts is recommended and compare the use rate of LLINs in different governorates with elucidation factors affecting use rate in different areas.

There is a need to study the effect of using LLINs on the prevalence of malaria cases among vulnerable groups (pregnant women and children under five years) by comparing cases of malaria among vulnerable groups before and after using LLINs.

\section{Conclusions}

In our study area the use rate of LLINs in general among vulnerable groups to malaria infection is relatively high; about three quarters of vulnerable groups (74.3\%) in the study area use LLINs.

The use of LLINs is strongly associated with having correct information about how to use and how to take care of LLINs, it is also associated with the educational status of housewives and educational status of fathers, but there is no significant association between the use of LLINs and type of house, the income of households, age and occupation of housewives, also there is no association with age and occupation of fathers.

There is no association between the use rate of LLINs and the residence of study population (urban, rural).

Almost two third of study population (65.2\%) recognized mosquito bite as a route of malaria transmission.

High percentage of study population (84.9\%) recognized high body temperature as a malaria symptom, but one third of them don't know any of malaria complications.

Less than half of the study population $44.4 \%$ know that using of LLINs is a protective method from malaria infection, while around one third of them (34.8\%) do not know any protective method from malaria infection. 
A high percent of the study population (84\%) have correct information about how to use and how to take care of LLINs; the main source of information was health workers who distributed them. There is limited role of radio and TV in the dissemination of information about LLINs.

Unfortunately the majority of study population didn't realize pregnant women as a vulnerable group to malaria infection, only $13.4 \%$ mentioned pregnant women, but more than three quarters $(77.9 \%)$ realized children are the vulnerable group to malaria infection.

The main reason for using LLINs among households (84.8\%) is a protection from nuisance of insects rather than malaria prevention. The main three reasons of not using LLINs (62.9\%) are: there are no mosquitoes and other insects, LLINs need fixation and chemicals cause allergy.

\section{Recommendations}

There is a need to increase health awareness about malaria infection and LLINs in malaria endemic areas in reproductive health clinics especially antenatal and immunization clinic to increase health awareness about malaria infection and the vulnerable groups to malaria infection especially pregnant women because the results of this study showed that the majority of the study population didn't realize pregnant women as vulnerable group to malaria infection and to focus on mothers who are responsible for using LLINs among vulnerable groups to malaria infection. This health education should include the route of malaria transmission which is important information for using protective methods to malaria infection as LLINs, because findings of this study revealed that there is some shortage of information about the route of malaria infection, also increase health awareness about LLINs in areas of LLINs distribution, this information not only about how to use and take care of LLINs but also about the role of LLINs in malaria prevention especially for vulnerable groups to malaria infection, and explain the easier way to fix LLINs and the safety of chemicals used in treating them.

Conduct periodically health education campaigns to improve health education regarding malaria infection and the role of LLINs in malaria prevention in areas of LLINs distribution either in schools or places of people's meeting as weekly markets in villages to target men and women associations. Health education campaigns should be accompanied with LLINs distribution and it can be associated with immunization campaigns.

Because there is a low contribution of media in health education about LLINs, there is a need to strengthen health education program about LLINs; advantages in malaria prevention, Proper use, keeping and vulnerable groups targeted by LLINs to broadcast through different media especially local Radios.

\section{Conflicts of Interest}

The authors declare no conflicts of interest regarding the publication of this paper. 


\section{References}

[1] World Health Organization (2005) What Is Malaria (Quick Overview). Regional office for the Eastern Mediterranean, Roll Back Malaria. World Health Organization, Geneva.

[2] Global Fund to Fight AIDS, Tuberculosis and Malaria (2004) The Status and Impact of Three Diseases. Malaria 2004. Global Fund to Fight AIDS, Tuberculosis and Malaria, Geneva, 4-39.

[3] National Center for Zoonotic Division of Parasitic Diseases (2004) Vector-Borne and Enteric Diseases, Malaria.

[4] Ministry of Public Health and Population (MoPH\&P) and National Malaria Control Program (NMCP) (2004) Annual Report 2004. Ministry of Public Health and Population, Sana'a, 3-5.

[5] Bin Ghouth, A. (2001) Malaria in Hajjar Valley School Malariometry: Environmental, Social and Health Indicators. Al-Mukalla, 3.

[6] Center for Malaria Researches, Ministry of Public Health and Population of Yemen (2000) Plan of Action. Unpublished Paper, Ministry of Public Health and Population of Yemen, Abyan.

[7] National Malaria Control Program (NMCP) (2003) Annual Report. National Malaria Control Program, Sana'a, 23-25.

[8] Al-Maktari, M.T. and Bassiouny, H.K. (1999) Bionomics of Anophiline Vectors in Zabid District, Al-Hodieda Governorate, Republic of Yemen. Eastern Mediterranean Health Journal, 5, 698-705.

[9] National Malaria Control Program (NMCP) (2005) The Third National Seminar on Malaria \& Other Vector Born Diseases VBDs. National Malaria Control Program, Sana'a, 40-43.

[10] National Malaria Control Programme (NMCP) (2006) Annual Report of Al-Hodieda Governorate 2005. National Malaria Control Programme, Tihama Region, Republic of Yemen, 2-5.

[11] World Health Organization (2000) Malaria in Yemen: Situational Analysis and Objectives. Technical Report. World Health Organization Regional Office for the Eastern Mediterranean (Yemen), Sana'a.

[12] Central Statistics Organization (2005) Population Census December 2004. Ministry of Planning \& International Cooperation, Republic of Yemen.

[13] World Health Organization (2002) Introduction to Basic Epidemiology and Principles of Statistics for Tropical Diseases Control. Communicable Diseases Cluster Department of Control, Prevention and Eradication Social Mobilization and Training Unit. World Health Organization, Geneva.

[14] Noor, A.M., Omumbo, J.A., Amin, A.A., Zurovac, D. and Snow, R.W. (2006) Wealth, Mother's Education and Physical Access as Determinants of Retail Sector Net Use in Rural Kenya. Malaria Journal, 5, Article No. 5. https://doi.org/10.1186/1475-2875-5-5

[15] Njama, D., Dorsey, G., Guwatudde, D., Kigonya, K., Greenhouse, B., Musisi, S. and Kamya, M.R. (2003) Urban Malaria: Primary Caregivers' Knowledge, Attitudes, Practices and Predictors of Malaria Incidence in a Cohort of Ugandan Children. Tropical Medicine and International Health, 8, 685-692.

https://doi.org/10.1046/j.1365-3156.2003.01060.x

[16] Osero, J.S., Otieno, M.F. and Orag, A.S. (2005) Maternal Use of Insecticide-Treated Nets in the Prevention of Malaria among Children under Five Years in Nyamira 
District, Kenya. East Africa Medical Journal, 82, 495-500.

https://doi.org/10.4314/eamj.v82i10.9343

[17] Adongo, P.B., Kirkwood, B. and Kendall, C. (2005) How Local Community Knowledge about Malaria Affects Insecticide-Treated Net Use in Northern Ghana. Tropical Medicine \& International Health, 10, 366-378.

https://doi.org/10.1111/j.1365-3156.2005.01361.x

[18] Okrah, J., Traore, C., Pale, A., Sommerfeld, J. and Müller, O. (2002) Community Factors Associated with Malaria Prevention by Mosquito Nets: An Exploratory Study in Rural Burkina Faso. Tropical Medicine and International Health, 7, 240 248. https://doi.org/10.1046/j.1365-3156.2002.00856.x

[19] Gyapong, M., Gyapong, J.O., Amankwa, J., Asedem, J. and Sory, E. (1996) Introducing Insecticide Impregnated Bednets in an Area of Low Bednet Usage: An Exploratory Study in North-East Ghana. Tropical Medicine \& International Health, 1, 328-333. https://doi.org/10.1046/j.1365-3156.1996.d01-41.x

[20] Rhee, M., Sissoko, M., Perry, S., McFarland, W., Parsonnet, J. and Doumbo, G. (2005) Use of Insecticide-Treated Nets (ITNs) Following a Malaria Education Intervention in Piron, Mali: A Control Trial with Systematic Allocation of Households. Malaria Journal, 4, Article No. 35. https://doi.org/10.1186/1475-2875-4-35

[21] Nganda, R.Y., Drakeley, C., Reyburn, H. and Marchant, T. (2004) Knowledge of Malaria Influences the Use of Insecticide Treated Nets but Not Intermittent Presumptive Treatment by Pregnant Women in Tanzania. Malaria Journal, 3, Article No. 42. https://doi.org/10.1186/1475-2875-3-42

[22] Onwujekwe, O., Uzochukwu, B., Ezumah, N. and Shu, E. (2005) Increasing Coverage of Insecticide-Treated Nets in Rural Nigeria: Implications of Consumer Knowledge, Preferences and Expenditures for Malaria Prevention. Malaria Journal, 4, Article No. 29. https://doi.org/10.1186/1475-2875-4-29

[23] Ahmed, N. and Matta, S. (1986) Annual Description Report for the Year 1986. Malaria Control Project. Yemen Unpublished WHO Report, World Health Organization, Geneva. 